\title{
Circadian rhythm of peak expiratory flow in asthmatic and normal children
}

\author{
A J W HENDERSON, F CARSWELL \\ From the Respiratory Research Group, Institute of Child Health, Royal Hospital for Sick Children, Bristol
}

\begin{abstract}
A study was undertaken to examine the circadian rhythm of peak flow rate in asthmatic and normal children in the community by means of cosinor analysis. An initial study of 12 matched pairs of asthmatic and normal children was used to determine the mean amplitude of peak expiratory flow (PEF) variability in the two groups $(7.8 \%$ and $4.4 \%)$ and the number of subjects required to show a significant difference. On the basis of this study 37 community based children with asthma diagnosed by questionnaire and 40 control subjects measured PEF four times daily for 14 days. Cosinor analysis of the data produced a significant fit in 20 asthmatic and 18 control children. A small but significant difference in amplitude was observed between the asthmatic $(6 \cdot 2 \%)$ and the control $(4.2 \%)$ children. There was no significant phase difference between the rhythms in the two groups. Cosinor analysis explained $14 \%$ of PEF variance. It did not provide a reproducible estimate of phase between week 1 and week 2; the acrophase changed by more than one hour in 26 of the 37 asthmatic children. The cosinor model may be inappropriate for the investigation of low amplitude circadian rhythms, especially when measurements are made infrequently.
\end{abstract}

\section{Introduction}

Many human biological systems, including airway calibre, show a circadian rhythm. This may be because the airways are passively influenced by the rhythm of related endogenous systems or because they respond directly to cyclically varying exogenous stimuli.

Cosinor analysis has been used to characterise the circadian rhythm of peak expiratory flow (PEF) in asthmatic and normal subjects.' Adult asthmatic patients have a rhythm of PEF variation that is in phase with the rhythm that may be detected in nonasthmatic control subjects but the amplitude of variation is greater. Two uncontrolled studies of asthmatic children have also shown a circadian rhythm in PEF on the basis of cosinor analysis. ${ }^{34}$ The rhythm in children was shown to be in phase with that described in adult asthmatic subjects with the bathyphase occurring between 24.00 and 08.00 hours. The proportion of children in whom a rhythm could be detected and the mean amplitude of the rhythm were, however, dissimilar in the two studies. This may be due to selection

Address for reprint requests: Dr F Carswell, Institute of Child Health, Royal Hospital for Sick Children, Bristol BS2 8BJ.

Accepted 3 February 1989 of patients as one series looked at hospital outpatients ${ }^{4}$ and the other at a community sample of children whose asthma was diagnosed by questionnaire. The amplitude of PEF variation increases around the time of an acute exacerbation of asthma in adults, ${ }^{2}$ and this may account for the larger amplitude of rhythms detected in asthmatic patients in hospital convalescing from acute asthma. As might therefore be predicted, the amplitude of PEF circadian rhythm has been shown to correlate with mean PEF. ${ }^{4}$

It is not therefore clear whether the differences observed in the circadian rhythm of PEF reflect a fundamental difference in the biological rhythm of airways calibre between asthmatic and non-asthmatic subjects or whether the differences are related to changes associated with a recent attack of asthma. The present study examines the PEF circadian rhythm in clinically stable asthmatic children and non-asthmatic control subjects to determine whether asthma is characterised by a fundamental difference in the $\stackrel{?}{?}$ amplitude or phase of PEF rhythm.

\section{Methods}

The studies were approved by Bristol and Weston Hospitals ethical committee. 
PEF was recorded with mini Wright peak flow meters (Clement Clarke International, United Kingdom). These were calibrated against constant flows $(100,200,300$, and $400 \mathrm{l} / \mathrm{min})$ before and after the study. No meter was accepted if the reading deviated by more than $10 \%$ from the measured flow. The coefficient of repeatability of the meters used in this study was $5 \mathrm{l} / \mathrm{min}$. Each child given a peak flow meter was instructed in its use. All measurements were made with the child in the upright posture and recorded as the best of three maximal blows into the peak flow meter. Six asthmatic children made three consecutive PEF measurements every hour for 24 hours. The coefficient of repeatability of three PEF measurements in this group was $42 \mathrm{l} / \mathrm{min}$.

The data were analysed by normalising PEF measurements to percentage deviation from the observed mean. A sine curve was approximated to the data by least squares regression on the basis of Halberg's cosinor method. ${ }^{5}$ The model assumes a period of 24 hours and the equation can be solved to yield values of the mesor, the amplitude, and the phase of the fitted curve. Amplitude is expressed as percentage deviation from the mesor. Goodness of fit of the sine function was assessed by the use of an $F$ test of the variance ratio. ${ }^{6}$ Amplitude was also calculated as the difference (D) between the highest and the lowest daily $P E F$ reading as a percentage of the daily mean (M) PEF $(\mathrm{D} / \mathrm{M} \times 100 \%)$.

In a pilot study six children with stable asthma drawn from an asthma clinic and five control subjects measured PEF hourly for two separate 24 hour periods. The cosinor model explained an average of $35 \%$ of the PEF variance in these children and fitted the data significantly in five of eleven 24 hour records from the asthmatic subjects and in seven of nine 24 hour records from the control subjects.

All other studies were carried out in a sample of children from the community, a questionnaire being used to diagnose asthma. ${ }^{7}$ Control subjects were taken from the non-asthmatic population without reference to other atopic features.

\section{PILOT STUDY}

Analysis of replies to a questionnaire from 300 pupils aged 7-11 years in one Bristol primary school identified 19 children with asthma. These were matched for sex, school class, and age with 19 non-asthmatic respondents. Each pair of children measured PEF at the same 29 set times over nine days. The times were chosen so that PEF was measured at four hourly intervals during each of two consecutive weekends and at eight hourly intervals during the intervening week.

Power analysis of these data was used to predict the numbers required for a larger study to ensure that a small amplitude difference between asthmatic and control subjects would be detected.

The study was carried out in June and July 1987, a time that includes the Bristol grass pollen season. One asthmatic patient had previously been diagnosed as having grass pollen allergy (a further five having symptoms of allergic rhinitis in the pollen season).

\section{MAIN STUDY}

The asthma questionnaire was sent to 800 pupils in three Bristol primary schools. Fifty asthmatic children were identified. Fifty non-asthmatic children from the remaining pupils were matched with them to act as controls.

The children were asked to measure their PEF four times daily for a predetermined two weeks. They were instructed to make one PEF measurement on waking and one on retiring plus two other measurements at intervals during the day. The times chosen on day 1 were used for all subsequent study days. The children were also asked to record day time symptoms, nocturnal symptoms, and symptomatic bronchodilator use on an asthma diary card. The school nurse provided an independent record of absences due to respiratory illness in the previous term for each child taking part in the study.

The data were collected in February and March 1988.

\section{ANALYSIS}

The goodness of fit of the PEF data (that is, the deviations from individual means) to a cosinor model was tested by the $F$ ratio. The amplitude of the deviations was normally distributed in the asthmatic and control groups. Amplitude was compared in the matched pair children by Student's paired $t$ test and in all other groups by the non-paired $t$ test. Acrophase was compared in the matched pair children by the signed rank sum test and in the other children by the Mann-Whitney $U$ test. Comparisons of symptoms and days off school were by the Mann-Whitney U test.

\section{Results}

\section{PILOT STUDY}

Twelve pairs (five male, seven female) of asthmatic and control children completed the pilot study. The mean (SD) age of the asthmatic children was 10 years 1 month $(8 \mathrm{~m})$ and of the control children 9 years 10 months $(8 \mathrm{~m})$. The pairs were completely matched for school class and sex. None of the control children was taking medication. Five of the asthmatic children were taking beta agonists as required and four were having regular prophylactic treatment (two slow release theophylline, one cromoglycate, and one inhaled corticosteroids). Three were having no treatment. The mean amplitude derived from cosinor analysis 
(percentage deviation of PEF measurements from the mesor) was $7.8 \%$ for the asthmatic subjects and $4.4 \%$ for the control group $(p=0.08)$. When amplitude was taken as the difference between the highest and the lowest reading of the day as a percentage of the day's mean the differences were significant $(18.4 \% v 9.6 \%$; $\mathrm{p}<0.02$ ).

\section{MAIN STUDY}

Thirty seven of the asthmatic and $\mathbf{4 0}$ control subjects successfully completed the main study. The asthmatic subjects consisted of 16 girls and 21 boys (mean age 9 years 10 months) and the control subjects consisted of 19 girls and 21 boys (mean age 9 years 6 months). None of the control subjects was taking medication. Eleven of the asthmatic subjects were receiving no treatment for their chest, 14 were taking beta agonists as required, and nine were receiving regular treatment (six inhaled steroids, two slow release theophylline, and one a combination).

The children with asthma had greater disability than the non-asthmatics in terms of day time or nocturnal symptoms. The median (range) of day time symptoms for the two weeks (total possible 112) was 15(0-57) in the asthmatic children compared with $0(0-27)$ in the control children $(p<0.001)$; the median nocturnal symptom scores (total possible 42 ) were $1 \cdot 5(0-23)$ and $0(0-6)(p<0.001)$. The asthmatic children also had more days off school in the preceding term $(5$ (0-23) days) than the control subjects $(2(0-13)$ days; $\mathrm{p}=0.02)$. There was no significant difference in mean PEF as a percentage of that predicted from height ( $96 \%$ in the asthmatic subjects, $106 \%$ in the control subjects).

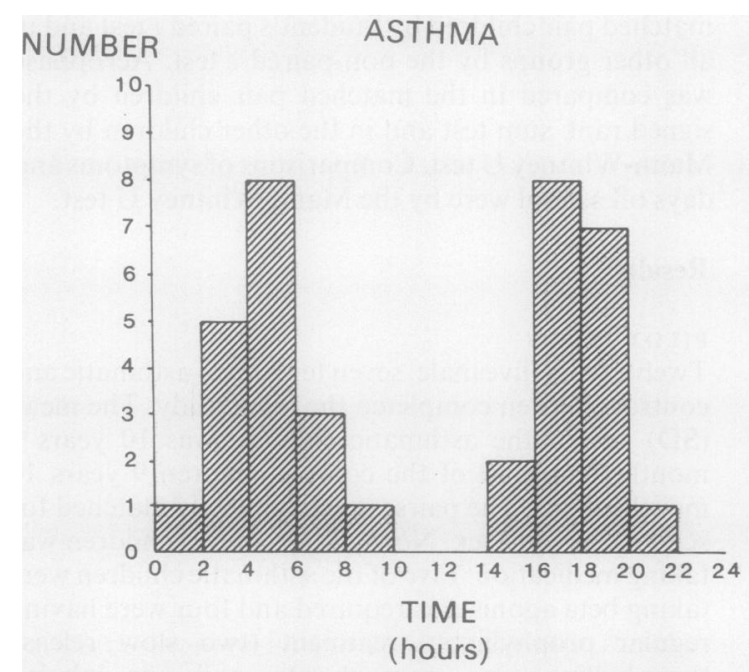

Amplitude of PEF circadian rhythm

Twenty of the 37 asthmatic PEF records and 18 of the 40 control PEF records fitted the cosinor model significantly ( $F$ test, $p<0.05$ ). The model explained $14 \%$ of the PEF variance in the main series. The mean (SEM) amplitude of variation derived from cosinor analysis was $6.2 \%(0.6 \%)$ in the asthmatic subjects and $4.2 \%(0.4 \%)$ in the control subjects. The cosinor amplitude was higher in the asthmatic subjects $(p<0.01)$.

When amplitude was calculated as the difference between extreme readings as a percentage of the mean the values were greater in the asthmatic than the control subjects $(12.5 \% \vee 8.8 \% ; p<0.02)$.

The amplitude of circadian variation was greater in the asthmatic subjects taking bronchodilator drugs as required (mean (SEM) $7.8 \%(0.9 \%)$ than in those having no treatment (mean $4.1 \%(0.9 \%) ; p<0.01)$. There were no differences in mean amplitude between the patients receiving regular treatment $(5.9 \%(1.6 \%))$ and the other two groups. No significant correlations were found between the amplitude of PEF circadian rhythm and the subjective day and night time symptoms or the number of days off school for illness in the preceding school term.

\section{Phase of PEF circadian rhythm}

In the main study the distribution of the acrophases in the asthmatic and control groups was bimodal Almost as many asthmatic and control subjects had a peak between 24.00 and 08.00 hours (nocturnal peak) as had a peak between 12.00 and $20.00 \mathrm{~h}$ (nocturnal dip) (fig 1). The distribution of the acrophase was not dependent on the goodness of fit of the sine approxi-

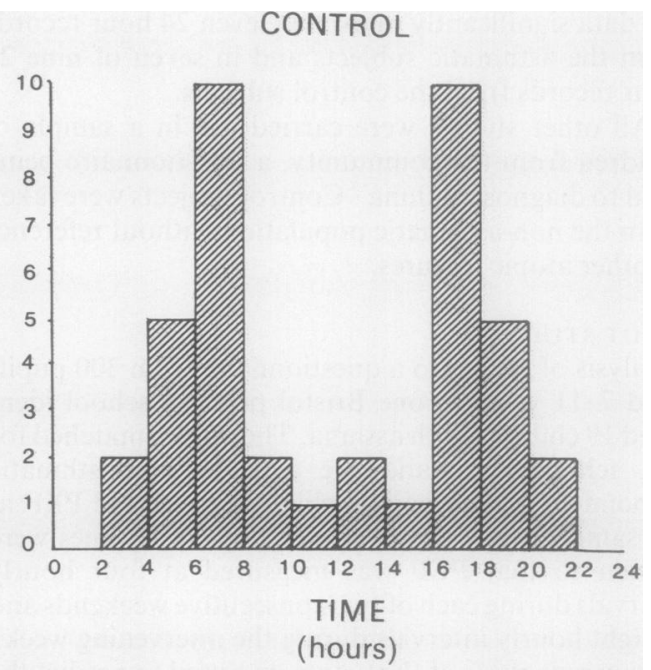

Fig 1 Distribution of acrophase estimates in the circadian rhythms of peak expiratory flow of 37 asthmatic and 40 control children. 


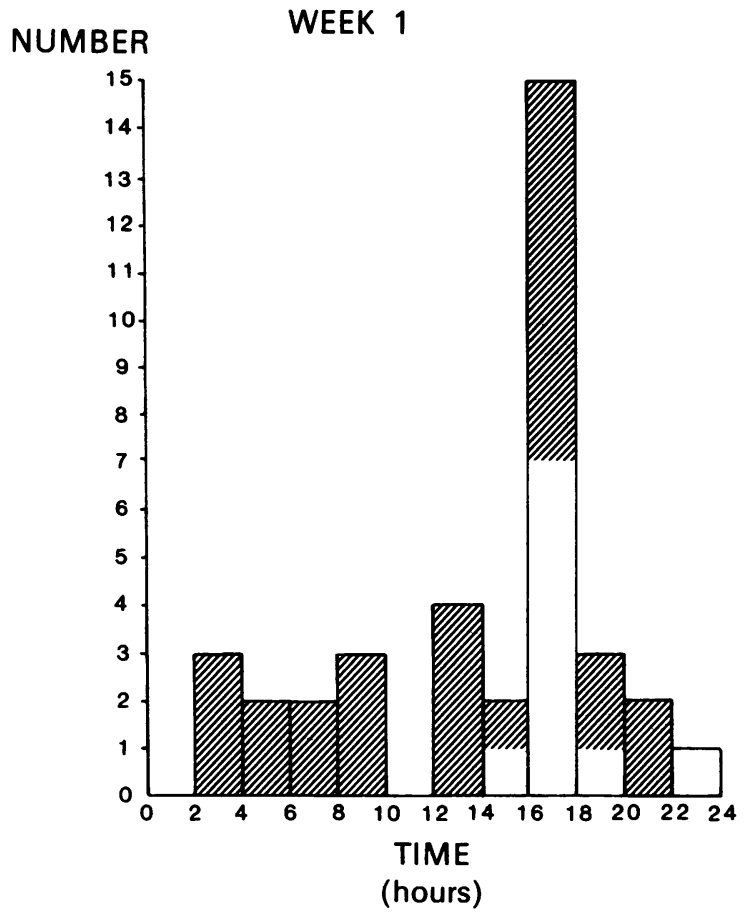

WEEK 2

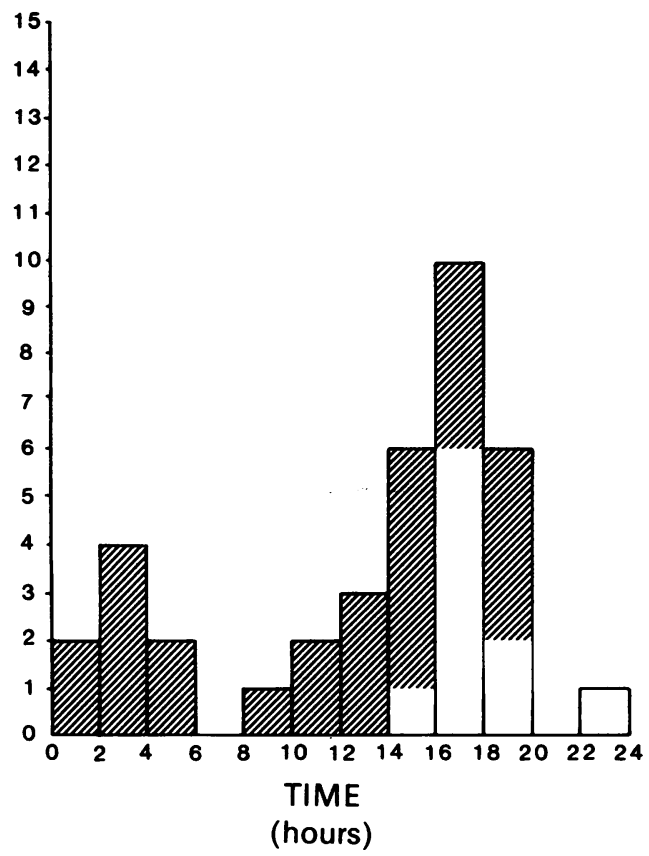

Fig 2 Distribution of acrophase estimates in the circadian rhythms of peak expiratory flow of 37 asthmatic children calculated separately for week 1 and week 2 of the study. The 10 subjects in whom a phase shift of less than 60 minutes occurred are indicated by the white portions of the columns.

mation as the distribution showed a similar bimodal pattern when the non-significant rhythms were excluded. There was no significant difference in the median acrophase between the two groups $(p=0 \cdot 6)$.

There were no differences in daytime or noctural symptoms between the asthmatic subjects with a predicted acrophase between 24.00 and $12.00 \mathrm{~h}$ and those with a predicted acrophase between 12.00 and $24.00 \mathrm{~h}$.

Each of the two week PEF records was analysed independently by cosinor analysis to assess the within subject reproducibility. There was no significant difference in amplitude between the first and second weeks, the mean difference being $1.89 \%$. There was also no significant difference in the median acrophase in week $1(17.25 \mathrm{~h})$ and week $2(14.59 \mathrm{~h})$. In only 10 of the 37 subjects, however, was the phase within one hour of that found in the previous week; 10 had a phase shift of 1-6 hours and 12 had a phase shift of 6-12 hours (fig 2).

\section{Discussion}

Using a sinusoid model of circadian rhythm of peak flow, we have found a significantly greater amplitude in a community sample of asthmatic children than of non-asthmatic control subjects. The amplitude of variation observed in this study was lower than that previously described in asthmatic children ${ }^{34}$ and the difference between the group mean amplitudes was smaller than that reported in adults. ${ }^{1}$ Several factors may have contributed to this finding. Our community population, like that observed by Johnston and others, ${ }^{3}$ is likely to have included a greater proportion of children with mild asthma than hospital based populations. The amplitude of PEF circadian variation is increased after an acute exacerbation of asthma ${ }^{89}$ and this may explain the large amplitudes reported in adult patients convalescing from acute asthma. ${ }^{1}$ This phenomenon may be related to an increase in bronchial hyperreactivity ${ }^{10}$ in addition to a reduction in mean $\mathrm{PEF}^{4}{ }^{4}$ The community basis of our study may have led to the identification of more asthmatic children with less substantial bronchial reactivity and a high baseline PEF, who would therefore be expected to have a lower amplitude of PEF circadian rhythm than patients with a recent exacerbation of their asthma.

One of the advantages of cosinor analysis over simpler mathematical models is that the phase of the 
rhythm can be calculated. In keeping with other studies of PEF variability, ours has found no difference in phase between the rhythms of asthmatic and control subjects. The expected bathyphase is reported to occur between the hours of 24.00 and 08.00 hours, which was the case in about half of each group in our study. The bimodal distribution of the acrophases was unexpected and is not explained by the exclusion of data that provided a poor fit to a sine curve (included in fig 1 ).

Cosinor analysis was selected for this study as it offers an attractive method of identifying predictable rhythms within a time series of unequally spaced data within which a large amount of biological noise might be expected. It has been widely used in studies of lung function in adults and children with asthma, though we are not aware of a controlled study of children using this method. There are theoretical reasons for reservations about its use. PEF variability results from a complex interaction between the effect of various endogenous rhythms, such as corticosteroid release," on airway function and direct and indirect exogenous stimulation. A simple mathematical formula is perhaps unlikely to describe the output of such an interaction accurately. Direct inspection of PEF changes with time suggests that the variation is not sinusoidal but that a rapid fall occurs at night with a slow rise during the day.

There are also practical objections. The overall fit of the curve was poor when measurements were made four times daily and the cosinor model explained only $14 \%$ of the PEF variance. The fit may be improved by more frequent measurements, such as the hourly measurements made in our pilot study, but there are practical problems in carrying out such frequent measurements, especially in children. The lower frequency of measurements made in our larger series is likely to have reduced the ability of the cosinor model to detect rhythms of low amplitude in a noisy system. The inclusion of such poorly fitting data in our analysis of amplitude may have lowered the group mean values.

When we studied the one week periods separately, we observed poor repeatability of acrophase estimates between the first and the second week. If the rhythm is a true, fundamental biological phenomenon we would not expect such rapid, large phase shifts in children with clinically stable asthma. Once again, observation of raw PEF data suggests that the true timing of peaks and troughs does vary from day to day; but the whole purpose of the mathematical model is to extract the predictable rhythmicity from such random background noise. With our schedule of measurements four times a day cosinor analysis does not appear to be adequate.
Cosinor analysis may not be a sufficiently sensitive tool for the routine investigation of low amplitude circadian rhythms of airway calibre. We have nevertheless detected circadian rhythms of PEF in both asthmatic and non-asthmatic schoolchildren. The difference in amplitude we observed seems likely to have been due to stimulation of the airways in the asthmatic children by exogenous factors, and if it were possible to observe asthmatic subjects with such stimuli completely excluded the amplitude of variation would probably be identical with that in normal subjects.

AJWH was supported by a grant from the British Lung Foundation. We are grateful to Ian Stewart of the Computer Centre, University of Bristol, for help with programming. We wish to thank all the children who took part in this project, as well as their parents and teachers, for their enthusiastic support.

\section{References}

1 Hetzel MR, Clark TJH. Comparison of normal and asthmatic circadian rhythms in peak expiratory flow rate. Thorax 1980;35:732-8.

2 Clark TJH, Hetzel MR. Diurnal variation of asthma. Br J Dis Chest 1977;71:87-92.

3 Johnston IDA, Anderson HR, Patel S. Variability of peak flow in wheezy children. Thorax 1984;39:583-7.

4 Sly PD, Hibbert ME, Landau LI. Diurnal variation of peak expiratory flow rate in asthmatic children. Pediatr Pulmonol 1986;2:141-6.

5 Halberg F, Engeli M, Hamburger C, Hillman D. Spectral resolution of low-frequency, small amplitude rhythms in excreted ketosteroid; probable androgen-induced circaseptan desynchronization. Acta Endocrinol 1965; suppl 103:1-54.

6 Nelson W, Tong YL, Lee J-K, Halberg F. Methods for cosinor rhythmometry. Chronobiologia 1979;6:305-23.

7 Carswell F, Brewin M, Hughes AO, Burr M, Carswell AM. Asthma in Avon schoolchildren [abstract]. Thorax 1985;40:694.

8 Hetzel MR. The pulmonary clock. Thorax 1981;36: 481-6.

9 Boulet L-P, Milot J. Comparison between the pattern of recovery of peak expiratory flow rates and symptom scores in acute exacerbations of asthma [abstract]. J Allergy Clin Immunol 1988;81:204.

10 Ryan G, Latimer KM, Dolovich J, Hargreaves FE. Bronchial responsiveness to histamine: relationship to diurnal variation of peak flow rate, improvement after bronchodilator and airway calibre. Thorax 1982; 37:423-9.

11 Soutar CA, Costello J, Ijaduola O, Turner-Warwick M. Nocturnal and morning asthma. Relationship to plasma corticosteroids and response to cortisol infusion. Thorax 1975;30:436-40. 